\title{
A Modified Adaptive Stochastic Resonance for Detecting Faint Signal in Sensors
}

\section{Qi Huang ${ }^{1}$, Jun Liu ${ }^{2, *}$ and Hengwei $\mathrm{Li}^{3}$}

1. Modern Industrial Design Institute, Zhejiang University, 38 ZheDa Road, Hangzhou 310027, China

2. Department of Biomedical Engineering, Zhejiang University, 38 ZheDa Road, Hangzhou 310027, China; E-mail: junliu@mail.bme.zju.edu.cn (Jun Liu)

3. Research Center of Language and Cognition, Zhejiang University, Hangzhou 310027, China

* Author to whom correspondence should be addressed. Fax: +86 571 87951676; E-mail: junliu@mail.bme.zju.edu.cn

Received: 16 July 2006 / Accepted: 5 February 2007 / Published: 15 February 2007

\begin{abstract}
In this paper, an approach is presented to detect faint signals with strong noises in sensors by stochastic resonance (SR). We adopt the power spectrum as the evaluation tool of SR, which can be obtained by the fast Fourier transform (FFT). Furthermore, we introduce the adaptive filtering scheme to realize signal processing automatically. The key of the scheme is how to adjust the barrier height to satisfy the optimal condition of SR in the presence of any input. For the given input signal, we present an operable procedure to execute the adjustment scheme. An example utilizing one audio sensor to detect the fault information from the power supply is given. Simulation results show that the modified stochastic resonance scheme can effectively detect fault signal with strong noise.
\end{abstract}

Keywords: faint signals, strong noises, stochastic resonance, bistable equation.

\section{Introduction}

Noises usually play a negative role on the detection of useful signals, and faint signal detection is a challenge work, especially detecting faint signals submerged in strong noise. Traditional filter is used to separate the signals of interest and their noise by restraining noises rather than using of them. For example, Fourier transforms and Wavelet transforms are the widely used idea which performs the filtering operation by projecting the signal into another space, then transforms back into the original space. But the approaches from this idea are effective only when the signals and the noise are not 
strongly mixed with each other in frequency spectrum. The phase-locked loop (PLL) is a typical approach to detect the faint signals by coherency modulating and demodulating. However, there is the limitation to this approach that is required to provide the reference signal with the same frequency as the detected signal. Other approaches are the application of statistical ideas to filtering problems, such as Kalman filter can make use of imprecise data on a linear (or nearly linear) system with Gaussian errors to continuously update the best estimate of the system's current state[1]. Least mean squares (LMS) filter is the adaptive filter which adjusts its coefficients to minimize the mean-square error between its output and that of an unknown system[2]. Another adaptive filter to minimize the least squares criterion is the recursive least squares (RLS) filter [3] and the QR-RLS filter[4] which perform QR decomposition and RLS algorithm. These adaptive filters can provide satisfactory solutions to linear or nonlinear problems. However, they focus on model description, optimal process, algorithm convergence and decreasing complexity. The properties of the signals and their noises are not used as the participator to improving filtering process. Especially, the noises mixed in the signals are always considered as the boring and unvalued factor in these approaches. In this paper, different from this traditional idea, we will utilize the noises to enhance the intensity of signals by nonlinear energy transition mechanism. In addition, there is a drawback among these filters that the signals of interest will inevitably be weakened in the process of suppressing noises, especially when the frequencies of noises and faint signals are very close.

However, noises are not always suppressed passively. They can be utilized to suppress themselves. In some systems increasing the amount of ambient noise actually enhances (up to a certain point) the SNR through a complicated nonlinear cooperation between the system and detector. This effect, known to operate in neurons [5], lasers [6], and analogue electrical circuits [7], is called SR, which is a counter intuitive nonlinear phenomenon wherein transmission or detection of a signal can be enhanced by addition of a non-zero level noise [8-10]. The noise fluctuations might be stochastic (meaning totally random) but the detection of a desired signal can be maximized by tuning the noise.

For SR, most of the studies were carried out using a dynamical system with bistability, modelled by a double well potential. Here SR is realised due to the shuttling between the two stable states at the frequency of the subthreshold signal (i.e. faint signal) with the help of noise. When the signal (faint signal with noises) is applied into a SR system, the potential barrier height of bistable system is adjusted to meet the condition of SR phenomenon. If SR occurs, the output spectrum of SR system would produce a sharp peak at the faint signal frequency superimposed on a smooth background spectrum [11]. This means that the output SNR is improved and faint signal is enhanced. Naturally, the adjusting process can be used as a useful procedure to process the receiving signal from the sensor or sensor arrays which need enhance the sensitivity in noisy environment.

This paper discusses how to use SR to detect faint signals with strong noises. Based on the bistable system, we combine SR phenomenon and Fast Fourier Transform (FFT) spectrum analysis together to detect faint signal mixed with noises. Note that the approach presented here is different from the aforesaid adaptive filter in design idea. Though those adaptive filters are in reality a non-linear system and can solve the nonlinear filtering problem, the nonlinear system need be translated into the linear system by various skills in the actual application process. The approach in this paper uses directly the nonlinear mechanism to construct filter and avoids the linearization process. Finally, an adaptive filtering procedure is added to the detection scheme to provide the possibility of application. The 
procedure resembles an executable program and its performance only depends on the adjustment strategy and evaluation index for SR from the angle of real application. In this sense, our approach is not a well-defined numerical algorithm indeed and is only an application scheme.

\section{SR System}

\subsection{Description of SR system}

Occurrence of SR phenomenon need three indispensable conditions: (1) nonlinear system, e.g., bistable (or multistable) nonlinear system; (2) signals to be detected; (3) noises. The simplest SR system is the bistable system which is defined by nonlinear Langenvin equation:

$$
\frac{d x(t)}{d t}=-V^{\prime}(x)+\xi(t)
$$

Where $x(t)$ is the state of the SR system, $\xi(t)$ represents band-limited Gaussian white noise with zero mean $\left\langle\xi(t)>=0\right.$ and autocorrelation function $\left\langle\xi\left(t_{1}\right) \xi\left(t_{2}\right)>=2 D \delta\left(t_{1}-t_{2}\right)\right.$ at time $t_{1}$ and $t_{2}$ (where $\delta(t)$ represents impulse function and $D$ is noise intensity). $V(x)$ denotes the potential function which processes a nonlinear symmetrical characteristic of $V(x)=V(-x)$. If a periodic signal $A \cos 2 \pi f t$ is applied to the Eq. (1) as the signal to be detected, then Eq. (1) will become

$$
\frac{d x}{d t}=-V^{\prime}(x)+A \cos 2 \pi f t+\xi(t)
$$

Suppose

$$
V(x)=\frac{1}{4} b x^{4}-\frac{1}{2} a x^{2}
$$

where $a, b>0$, and then Eq. (1) will become

$$
\frac{d x}{d t}=-V^{\prime}(x)+A \cos 2 \pi f t+\xi(t)=a x-b x^{3}+A \cos 2 \pi f t+\xi(t)
$$

Eq. (4) describes an over-damped Brownian movement. The potential barrier of the system is defined as $\Delta V=a^{2} / 4 b$ when $A=0$ and $\xi(t)=0$. Referring to paper [12-14], we can know that there are two potential wells (stable points) when $x= \pm \sqrt{a / b}$ and there exists a critical value when the magnitude of input $A_{c}=\sqrt{4 a^{3} / 27 b}$.If the magnitude of the input signal is less than $A_{c}$ at a fixed potential barrier, then the periodic signal can not drive the bistable system to produce output response. As the noise $\xi(t)$ is added to the system and its intensity is enhanced gradually to a certain value, the total energy of signal and noise will possibly exceed $A_{c}$. On this condition, the output response will occur randomly due to transitions between two potential wells, and the output will also exhibit characteristic of the input signal according to a certain probability. On account of the cooperativity between signal and noise, there is the optimal noise intensity which can maximize the output response with the frequency $f$. Upon increasing the noise intensity, too many transitions are activated by noise during one cycle of the periodic input, and the cooperativity between signal and noise is lost again. This is the SR effect: the system's response is most regular at a finite, nonvanishing noise level. 


\subsection{Signal Processing by SR System}

According to the above discussion, the system described by dynamics equation $\frac{d x(t)}{d t}=-V^{\prime}(x)$ can be used to signal processing by SR. Note that the potential barrier in the system can be considered as the damp of the system. Therefore, the basic idea is to meet the condition of SR at the case of diverse inputs by modulating potential barrier. Further it can be realized by adjusting the parameter $a$ or $b$. Correspondingly, the critical values are also changed with the parameter adjustment.

The corresponding simulation example as follows is given to validate the effect of SR on detecting the electrical signal with the strong noise.

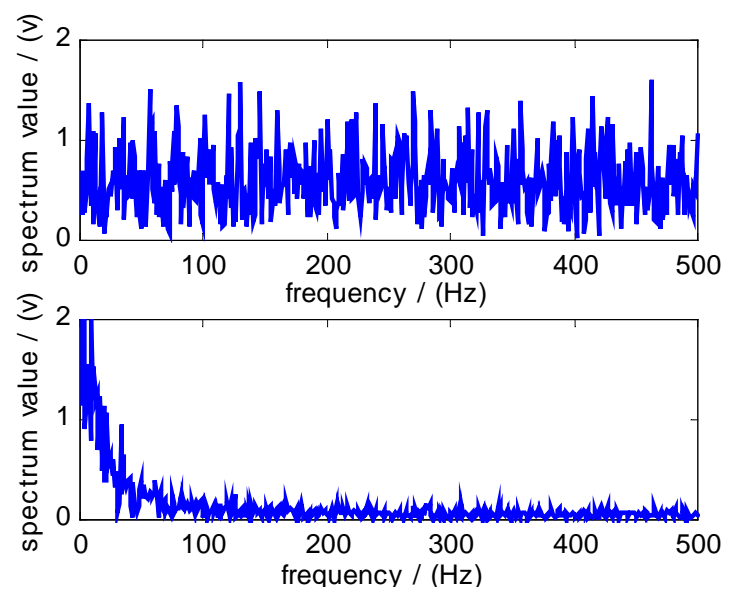

Figure 1. Input signal spectrum (upper figure) and output response spectrum (lower figure) of SR system when signal amplitude $A=0.1 \mathrm{~V}$, frequency $f=100 \mathrm{~Hz}$, noise intensity $D=0.5, a=6$ and $b=1$.

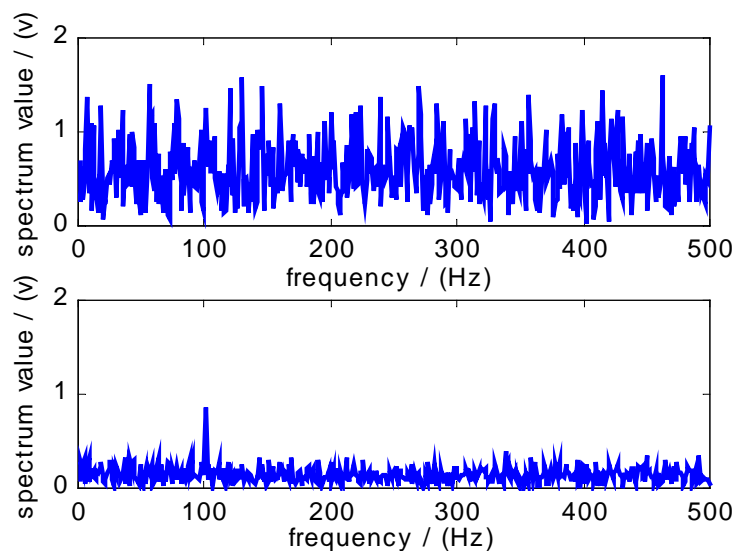

Figure 2. Input signal spectrum (upper figure) and output response spectrum (lower figure) of SR system when signal amplitude $\mathrm{A}=0.1 \mathrm{~V}$, frequency $f=100 \mathrm{~Hz}$, noise intensity $D=0.5, a=1$ and $b=1$.

Fig.1 and Fig.2 illustrate the influence of SR on detecting signal $A \cos 2 \pi f t$. The parameters relating with Fig. 1 are $a=6, b=1, A=0.1 \mathrm{~V}, f=100 \mathrm{~Hz}$ and the gaussian white noise intensity $D=0.5$.In term of these parameters and the aforementioned expression ( $\left.\Delta V=a^{2} / 4 b\right)$, the potential barrier $\Delta V=9$ is obtained. The parameters of Fig.2 are the same with those of Fig.1 except that the parameters $\mathrm{a}=1$, correspondingly, $\Delta V=0.25$. Here SR will occur (shown in Fig.2).

As shown in Fig.1 and Fig.2, the upper figure represents the input spectrum of signal with strong noise background, and the lower figure is the output spectrum of the SR system. In comparison with 
Fig.1, the frequency value $(100 \mathrm{~Hz})$ of signals can be obviously observed in Fig.2.The results indicate that the output signal spectrum is greatly improved via SR.

\section{Adaptive Filtering Procedure by SR}

For measurement of real signals, the pre-knowledge about signals is often lack; hence the abovementioned SR system can not be directly used. The practicable approach requires that the SR can adapt different input signals. Therefore, an adaptive stochastic resonance scheme based on bistable system is presented as follows.

We adopt the power spectrum as the evaluation tool of SR. Here the power spectrum can be obtained by the fast Fourier transform (FFT). Furthermore, we introduce the adaptive filtering scheme to realize signal processing automatically. The key of the scheme is how to adjust the barrier height to satisfy the optimal condition of SR in the presence of any input. A simple idea is to estimate the shift and rise of the power spectrum peak when the barrier height is modulated. If the spectrum peak at a certain frequency is significant relative to the rest frequency components, then we can conclude that SR occurs and the detected signal takes on this frequency feature.

Fig.3 shows the basic scheme which is composed of the adaptive filtering system. Firstly, the original signals from the transducer are applied to a SR system through the acquisition module where the preprocessing such as amplifying and filtering are completed. Then, the processed signals enter the adaptive part. In this part, SR system module can be represented by function (1). FFT module plays a role in evaluating the spectrum features of the output from SR system. In computing module, these spectrum features are used as the variable to estimate optimal condition. According to computing results, a regulating module is introduced to execute the feedback adjustment. On the collective effect of these modules, the parameter of SR system is gradually adjusted to the appropriate barrier potential and the critical value. When a system satisfies the condition of SR, the output contains the information of the detected signal.

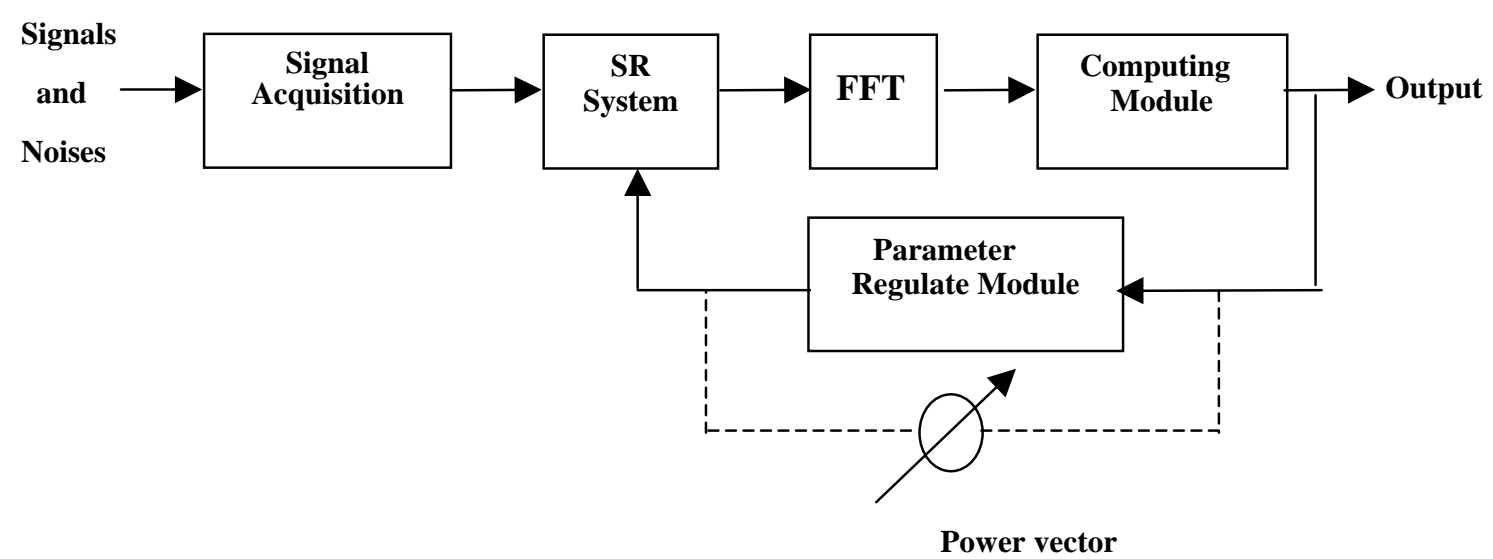

Figure 3. Scheme of signal detecting with SR system.

The signal noise ratio (SNR) is defined as the ratio of the spectrum value at a frequency $f_{i}$ (the frequency feature of input signal) to the mean spectrum value of the entire frequency range, respectively. It can be written as follows: 


$$
S N R=10 \log \frac{S\left(f_{i}\right)}{\operatorname{mean}\left[S\left(f_{1}\right), \ldots, S\left(f_{i}\right), \ldots .\right]}
$$

where $S\left(f_{i}\right)$ represents the spectrum of input signal, and mean $\left[S\left(f_{1}\right), \ldots, S\left(f_{i}\right), \ldots ..\right]$ represents the mean spectrum value. According to SR definition, a maximum value of $S N R$ will occurs with the increase of noise intensity in bistable system. This can be considered as the optimal condition for computing module. Note that SR is produced by adjusting the parameters of SR system in computing module since the input signal and noise is fixed. Though the procedure is different from the SR via changing the noise, the essential mechanism is uniform.

Our approach is not a well-defined numerical algorithm indeed and does not exist cost function or transfer function, although it resembles the control algorithm in the form of block diagram. Optimal condition is set up not according to mathematical deduction but according to phyical property of SR systmen. Therefore, we present only an application scheme as follow.

For the given input signal, we will present an operable procedure to execute the adjustment scheme. During this procedure, the optimal condition will be transformed into two inequation (5) and (6). If the spectrum features of detected signal satisfies the inequation (5) and (6), then it will be concluded that the signal is optimally detected by SR. The frequency component near the maximum value of SNR is estimated by the inequation (5).Further, the estimation precision is determined by the inequation (6). This inequation restricts the possible spectrum range and feature of the unknown signal. The parameter $\theta$ at the right of inequation (5) denotes the relative threshold which evaluates the significance of a certain frequency in power spectrum. Larger the relative threshold is, more precise the estimation value is.

$$
\begin{array}{r}
\text { estimation }_{f=f_{i}}=\frac{A_{f_{i}}-A_{\text {mean }}}{A_{f_{i}}} \geq \theta \\
P\left(f=f_{i}\right)=\frac{C_{f_{i}}}{C_{f_{1}}+C_{f_{2}}+\ldots . .+C_{f_{K}}} \geq \frac{1}{K}
\end{array}
$$

The estimation value for a certain frequency is expressed by the parameters $A_{f_{i}}$ and $A_{\text {mean }}$. The former represents the amplitude at a certain frequency $f_{i}$ and the latter is the mean amplitude of all frequency in power spectrum. The parameter $f_{i}$ is a frequency of power spectrum, $i=0,1, \ldots$, n. The parameter $C_{f_{i}}$ in inequation (6) denotes the amount of the case that estimation value for a frequency is greater than or equal $\theta$ at a series of power spectrums. Suppose $j$ the values of power spectrum to be taken, $C_{f_{i}}$ may be a value range from 0 to $j$. The parameter $K$ represents the amount of the different frequencies satisfying inequation (5). Therefore, $P\left(f=f_{i}\right)$ represents the probability that the significant frequency $f_{i}$ occurs relative to other frequency.

The detail of the procedure is described as follows:

Step1. For conveniently regulating the SR system, we change one of parameters in the system and fix the rest parameters. It is assumed that the parameter $b$ in equation (3) is constant. For example, it is taken as 1 . Correspondingly, we can adjust the potential barrier only by parameter $a$.

We take parameter $a$ as different values and observe whether SR system with the corresponding parameter $a$ induces the response. If there are responses of SR system for $m$ parameter $a$, then we will obtain $m$ spectrum values (e.g., $S_{1 \ldots} S_{m}$ ) by FFT module. Obviously, these spectrums have the same 
frequency range and resolution. Assuming each spectrum consists of $n$ points, then the values of frequency can be taken as $f_{0}, f_{1}, \ldots, f_{n}$.

Step2. Choose the appropriate threshold $\theta$. We can conclude from the inequation (5) that if the inequation is meaningful then threshold will be always less than one. We can also find the larger threshold can induce higher estimation precision. Therefore, we can restrict the threshold in a certain range. Here we set the initial value $\theta=0.95$.

Step3. For any frequency $f_{i}(i=0,1, \ldots, \mathrm{n})$ corresponding to a certain parameter $a$, we record the total number of which frequency amplitude satisfy inequation (5) at spectrum $S_{1}, S_{2}, \ldots, S_{m}$, and obtain the corresponding $C_{f_{i}}(\mathrm{i}=0,1 \ldots \mathrm{n})$ and $K$ according to inequation (6).

Step 4. Find out the parameter $f_{i}$ satisfying inequation (6) and continue the next step. If $f_{i}$ does not exist, then the parameter $\theta$ will be decreased. Return the step 3 .

Step5. If the threshold $\theta$ is less than or equal the given lower limit (e.g., 0.5), then the other parameter $a$ and its spectrum values will be considered. Return step2 .

Step6. For the parameter $a$, if the above two inequation is meaningful at limiting threshold range, we will conclude that the output reflect the feature of input signal via the SR effect. Recording the output and ending the procedure.

\section{Fault Test by Audio Sensor}

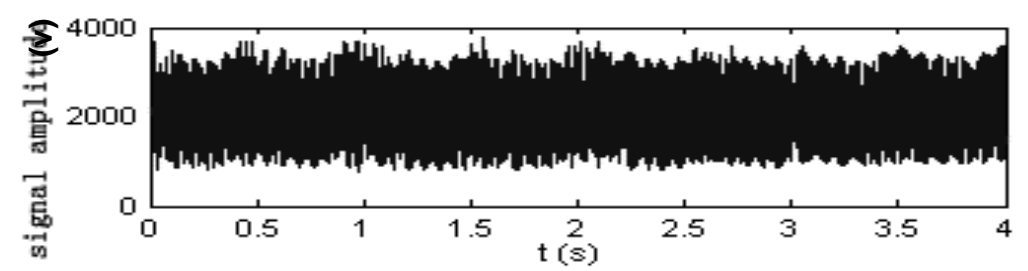

Figure 4. Audio signal of intermediate frequency power supply.

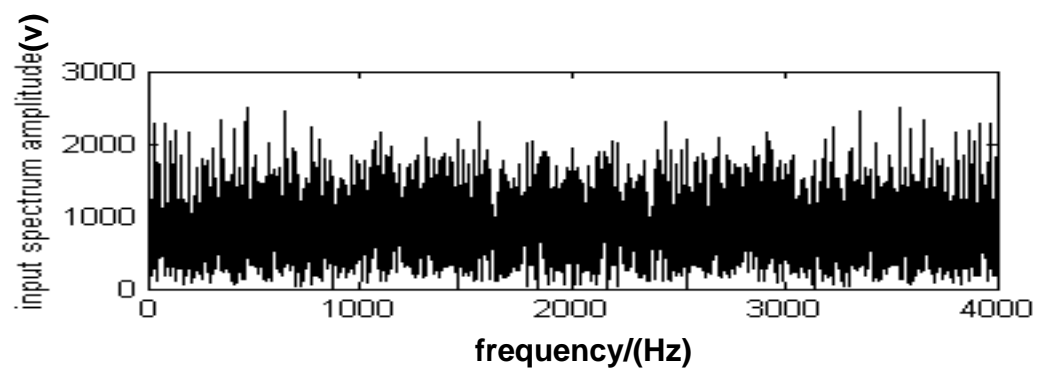

Figure 5. spectrum of input signal.

Figure 4 shows the audio signal from a power supply with fault, which is collected and transformed into electrical signal by the audio sensor. Here we use a electret test microphone as the audio sensor. For this microphone, frequency response ranges from $10 \mathrm{~Hz}$ to $5000 \mathrm{~Hz}$ and standard sensitivity is $25 \mathrm{mV} / \mathrm{Pa}$. It can be used as noise monitor for measure of ambient noise machine noise, vehicle noise, electrical machinery noise, architecture acoustics and electroacoustics.

In normal work status, a power supply emits the audio signal with range of frequency from 0 to $4000 \mathrm{~Hz}$. But the frequencies of fault signals, which directly relates with the degree of fault, are about tens of hertz. Obviously, "noise" is mixed with the fault signal to be detected. Fig. 5 shows the power 
spectrum of the signal in Fig.4. It can be found that the information relevant to fault cannot be directly distinguished by the power spectrum (as shown in Fig.5). In order to improve the sensitivity of fault detection, the adaptive SR approach has to be used to process the detected signal from audio sensor.

According to the abovementioned procedure, the fault signal with noise is considered as the input of the system in Fig.3. The parameter $\mathrm{b}$ is taken as the fixed value 1 and $\theta$ is equal to 0.9. The parameter $a$ is changed from 0.1 to 1 by the step length 0.1 , so the $m$ is set as 10 . Because the sample frequency is $500 \mathrm{~Hz}$, there are 2000 sample points during the signal of 4 seconds. Correspondingly, $\mathrm{n}$ is taken as 2000. The simulation of algorithm is performed by the simulation tool of Matlab software. After adjusting the parameter, the optimal SR can be obtained. Fig.6 shows the power spectrum of the corresponding output when SR occurs. The signals with frequency component $65 \mathrm{~Hz}$ and $80 \mathrm{~Hz}$ can be clearly recognize. According to experience, these signals belong to the fault information.

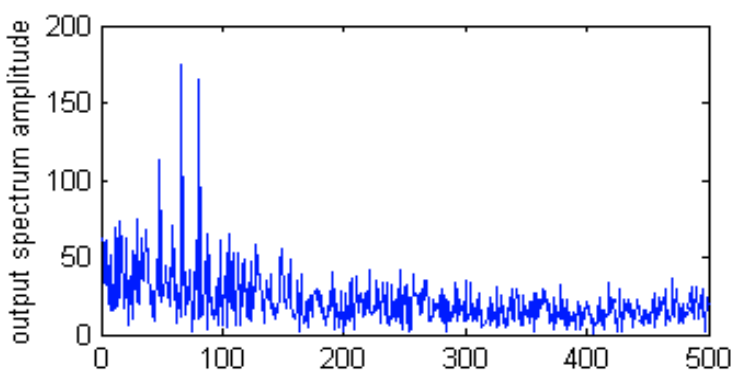

Figure 6. Frequency spectrum of output. The parameter is: $b=1 ; \theta=0.9 ; a=0.6$.

\section{Conclusion}

An application method of SR was presented by theoretical analysis and example simulation in this paper. We demonstrated the advantage of SR by comparing the signal processing results with and without SR. According to this idea, the adaptive filtering approach based on SR was given to improve signal processing conveniently. Correspondingly, the bistable equation was used as SR model, and power spectrum information was introduced as the evaluation index of the filter. An example utilizing audio sensor was used to detect the fault information from power supply. From frequency spectrum of the output, we can find the amplified spectrum feature of fault signal relative to the noise. This result indicates feasibility of the method. It is worth noting that if one SR system cannot realize the complex signal detection, i.e., optimal SR spectrum does not occur, second or more SR system may be used to obtain the optimal effect.

\section{Acknowledgements}

This work was partially supported by the National Natural Science Foundation of China (projects No. 30470470) and the Project of Zhejiang Province Education Department of China (20050907).

\section{References and Notes}

1. Hernandez, W. Improving the response of several accelerometers used in a car under performance tests by using Kalman filtering. Sensors 2001, 1, 38-52. 
2. Ferrara, E. Fast implementations of LMS adaptive filters. IEEE Transactions on Acoustics, Speech, and Signal Processing 1980, 28, 474-475.

3. Sayed, A.H.; Kailath, T. A state-space approach to adaptive RLS filtering. IEEE Signal Processing Magazine 1994, 11, 18-60.

4. Diniz P.; Siqueira, M. Analysis of the QR-RLS algorithm for colored-input signal. IEEE International Conference on Acoustics, Speech, and Signal Processing (ICASSP) 1995, 2, 14281431.

5. Bartussek, R.; Hänggi, P.; Jung, P. Stochastic resonance in optical bistable systems. Phys. Rev. E 1994, 49, 3930-3939.

6. Bulsara, A. R.; Jacobs, E. W.; Zhou, T.; Moss, F.; Kiss, L. Stochastic resonance in a single neuron model: theory and analog simulation. Journal of Theoretical Biology 1991, 152, 531-555.

7. Luchinsky, D.G.; McClintock, P.V.E. Irreversibility of classical fluctuations studied in analogue electrical circuits. Nature 1997, 389, 463-469.

8. Benzi, R.; Sutera, A.; Vulpiani, A. The mechanism of stochastic resonance. J. Phys. A 1981, 14, L453-L456.

9. Moss, F. Stochastic resonance in: a signal + noise in a two state system. Proceedings of the 45th Annual Symposium on Frequency control 1991, 649- 658.

10. Chapeau-Blondeau, F. Input-output gains for signal noise in stochastic resonance. Phys. Lett. A 1997, 232, 41-48.

11. Gingl, Z.; Vajtai, R.; Kiss, L.B. Signal-to-noise ratio gain by stochastic resonance in a bistable system. Chaos, Solitons \& Fractals 2000, 11, 1929-1932.

12. Fauve, S.; Heslot, F. Stochastic resonance in a bistable system. Phys. Lett. A 1983, 97, 5-7.

13. Lindner, J. F.; Mason, J.; Neff, J. Noninvasive control of stochastic resonance. Phys. Rev. E 2001, 63, 041107-041115.

14. McNamara, B.; Wiesenfeld, K. Theory of Stochastic Resonance. Phys. Rev. A 1989, 39, 4854 4869.

(C) 2007 by MDPI (http://www.mdpi.org). Reproduction is permitted for noncommercial purposes. 